\title{
Infrared and Visible Image Fusion Algorithm Based on Characteristic Analysis
}

\author{
Lu Xing-Hua \\ HuaLi College Guangdong University of Technology, 511325 \\ Guangdong Guang Zhou Zhengcheng, China \\ 44680189@qq.com
}

\begin{abstract}
There are existing fusion algorithm have some problems of Blur and the easy integration with the fusion effect. In response to this phenomenon, this paper presents an infrared and visible image fusion algorithm based on characteristic analysis. It is according the signal characteristics of the infrared and visible image to analysis the similarity of region. It is to classify processing the image signal in the last. The results show that the experimental results are basically consistent with the expected results. The fused image has the advantages of high definition and Anti-interference ability.
\end{abstract}

Keywords-fusion algorithm; infrared and visible images; characteristics; similarity

\section{INTRODUCTION}

Visible images have a clear outline of the image, but susceptible to interference. Infrared images often vague outline, but it can reflect target object in a bad case of the light. Therefore, In order to achieve complementary information, people is proposed fusion algorithm. Through it we can effective signal extraction of multi-image ${ }^{[1-3]}$. Such as reserved the clarity of visible image, reserved the infrared target from infrared image. Currently, The people made a number of fusion algorithm. Wherein the pixel-level fusion algorithm is the most. Such as those based Laplace decomposition, Wavelet transform Contourlet transform and nonsubsampled Contourlet Transform ${ }^{[4-6]}$.

Therefore, we are according the signal characteristics of the infrared and visible image. Presents an new infrared and visible image fusion algorithm. Article algorithm calculated similarity of image signal to classification the image signal. And it is according to the strength of correlation to classification processing the signal. When the correlation is stronger, this paper is using the intensity of the regional to processing. When the correlation is weak, we are using the fusion algorithm based on energy. It can effectively avoid loss of the valid signal. To validate the algorithm, we are using two sets of simulation images, which target is blocked. And we are using a variety of algorithms to compare. Those are in order to verify the feasibility of this algorithm. At last, The simulation results can be seen that proposed algorithm has advantage of strong anti-interference and high definition.

\section{PROPOSED ALGORITHM}

\section{A. Regional Similarity}

According to the characteristics of the infrared and visible image, we are calculated similarity of image signal ${ }^{[7-8]}$. Its functions are as follows.

$$
\begin{gathered}
\text { Similarity function: } \operatorname{Sim}\left(w_{t, A}, W_{t, B}\right)= \\
\frac{\left(2 \cdot W_{t, A}^{\prime \prime} \cdot W_{t, B}^{\prime \prime}+C_{1}\right)\left(2 \sigma_{W_{t, A}, W_{t, B}}+C_{2}\right)}{\left[\left(W_{t, A}^{\prime \prime}\right)^{2}+\left(W_{t, B}^{\prime \prime}\right)^{2}+C_{1}\right]\left(\sigma_{W_{t, A}}^{2}+\sigma_{W_{t, B}}^{2}+C_{2}\right)}
\end{gathered}
$$

Wherein $W_{t, A} 、 W_{t, B}$ represent the Wavelet coefficients of visible image A and infrared image $\mathrm{B}$. And $W_{t, A}^{\prime \prime}$ is the means of $W_{t, A}, \quad \sigma_{W_{t, A}}^{2}$ is the variance of $W_{t, A}$; And 
$\sigma_{W_{t, A}, W_{t, B}}$ is the covariance between off $W_{t, A}$ and $W_{t, B}$. Among them $C_{1} 、 C_{2}$ are the small constant factors.

The mean and variance in the domain are shown below.

$$
\text { Means: } w_{t, X}^{\prime \prime}=\frac{1}{m \times n} \sum_{i=1}^{m} \sum_{j=1}^{n} f(i, j)
$$

Variance: $\sigma_{w_{t, X}}^{2}=\frac{1}{m \times n} \sum_{i=1}^{m} \sum_{j=1}^{n}\left[f(i, j)-w_{t, X}^{\prime \prime}\right]^{2}$

\section{B. Classification}

When $\operatorname{Sim}\left(w_{t, A}, W_{t, B}\right) \geq \lambda_{2}$, In this case the similarity between regions is large. In order to better performed the integration processing. This paper is using the intensity of the regional to processing. The method is as follows.

By calculating the correlation of the image signal intensity, and using it to reflect the strength characteristics of source image window area signal. Its function is expressed as follows.

$$
\begin{gathered}
R_{j, A B}^{C}=I_{j, A}^{C} / I_{j, B}^{C} \\
I_{j, A}^{C}(i, j)=\sum_{i \in m, j \in n} P(i, j)\left|G_{A}(i, j)\right| \\
I_{j, B}^{C}(i, j)=\sum_{i \in m, j \in n} P(i, j)\left|G_{B}(i, j)\right|
\end{gathered}
$$

Wherein $G_{A}(i, j) 、 G_{B}(i, j)$ Respectively the detail components of the image A and image B. And Assume that the current detecting region at $\mathrm{C}$. Its size is $m \times n$. And $P(i, j)$ as a mask matrix, which is linear filter. In order to better fusion, we redefine the average gradient feature As follows.

The average gradient feature is the significantly different both sides of the border or cross hatching, I.e. the rate of variation in gray. The rate of change of this size can be used to represent the image sharpness. It reflects the small details contrast of the rate of change. Its function expression:
$G_{A}(i, j)=\frac{1}{m \times n} \sum_{i=1}^{m} \sum_{j=1}^{n} \sqrt{\frac{\Delta f_{x}^{2}(i, j)+\Delta f_{y}^{2}(i, j)}{2}}$

$G_{B}(i, j)=\frac{1}{m \times n} \sum_{i=1}^{m} \sum_{j=1}^{n} \sqrt{\frac{\Delta f_{x}^{2}(i, j)+\Delta f_{y}^{2}(i, j)}{2}}$

Wherein $G_{A}(i, j) 、 G_{B}(i, j)$ respectively the average gradient of the image $\mathrm{A}$ and image $\mathrm{B}$ in the low frequency area coefficient $\mathrm{C}(m \times n) \cdot \Delta f_{x}(i, j) 、 \Delta f_{y}(i, j)$

$\Delta f_{x}(i, j) 、 \Delta f_{y}(i, j)$ are respectively the difference value of the horizontal and vertical directions.

$$
\text { I.e. } \omega(i, j)= \begin{cases}\omega_{A}(i, j) & R_{j, A B}^{C} \geq 1 \\ \omega_{B}(i, j) & R_{j, A B}^{C}<1\end{cases}
$$

When $\operatorname{Sim}\left(W_{t, A}, W_{t, B}\right)<\lambda_{2}$, At this point of similarity between smaller areas, we are using the fusion algorithm based on energy. I.e. it is comparison with the amount of energy between image A and image B. When energy image A is greater than the image $\mathrm{B}$, The edge information a more significant. In contrast, it means the edge information B more significant. Its method is as follows.

$$
\omega(i, j)= \begin{cases}\omega_{A}(i, j) & E_{A}(\mathrm{x}, y) \geq E_{B}(\mathrm{x}, y) \\ \omega_{B}(i, j) & E_{A}(\mathrm{x}, y)<E_{B}(\mathrm{x}, y)\end{cases}
$$

\section{SIMULATION}

To test the algorithm effects of infrared and visible image fusion process. using MATLAB 2014a to test the algorithms. In order to get the better results, we using some algorithms to simulation test. Such as the image fusion algorithm based on wavelet transform, document algorithm ${ }^{[9]}$ and document algorithm ${ }^{[10]}$.

\section{A. Contrast Effects}

Choose the visible and infrared images, which fewer complexes and the target is covered. It is to testing fused image anti-jamming capability. And it is by adopting the 
above algorithms. Each algorithm was renderings as follows.

\section{B. First experiment}

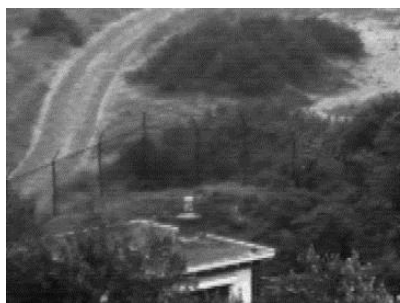

(a)visible image

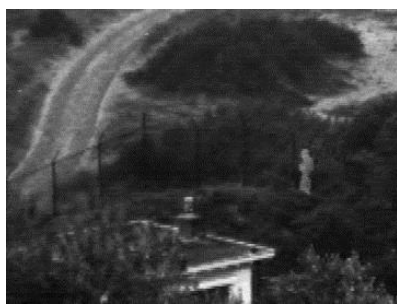

(c)Wavelet fusion algorithm

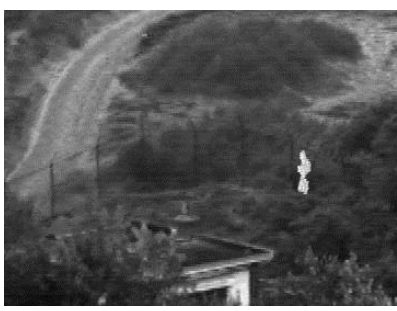

(e) Document algorithm [10]

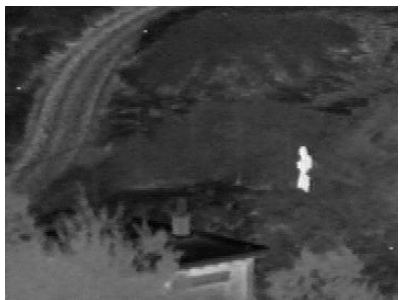

(b) infrared image

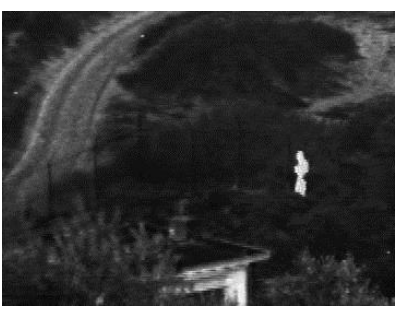

(d) Document algorithm [9]

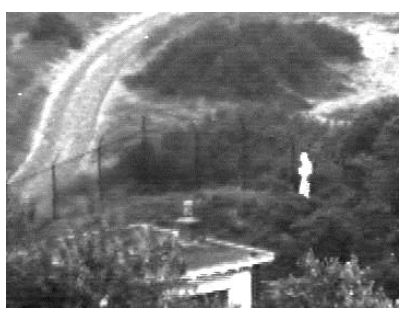

(f) Proposed method

Fig. 1 the integration renderings of the each algorithms

By observing each fusion algorithm renderings, we can be seen that proposed algorithm is better than other algorithms in fusion effect. Document algorithm [9] although can possible to obtain a good target signal, but it Ignore to protect the background signal. Document algorithm [10] is effective solving the problem of background Protection, but fusion mage is low contrast.

\section{Second experiment}

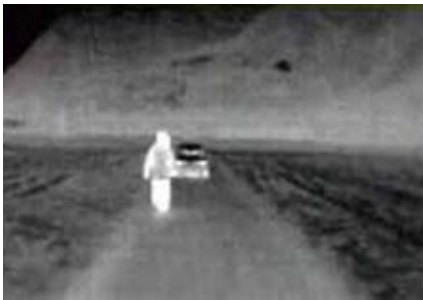

(a)visible image

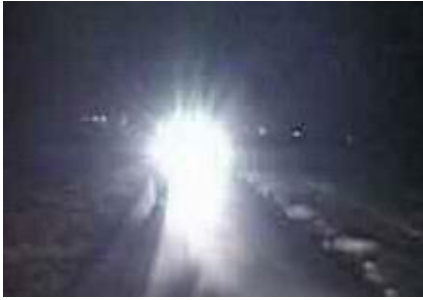

(b) infrared image

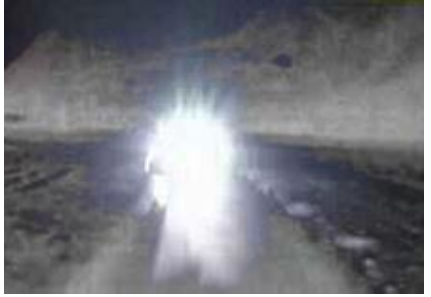

(c)Wavelet fusion algorithm

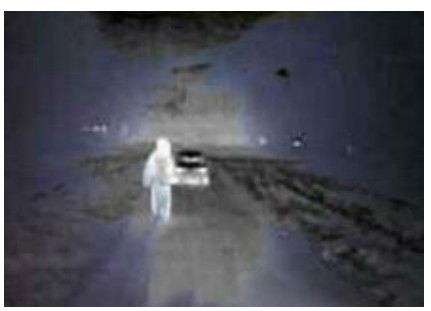

(e) Document algorithm [10]

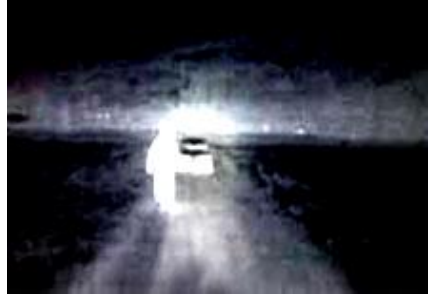

(d) Document algorithm [9]

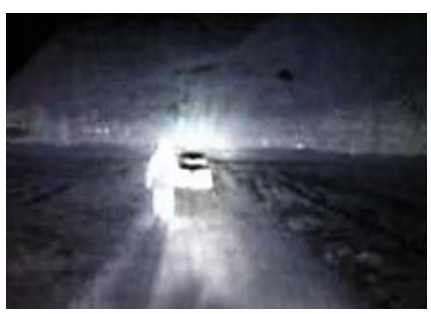

(f) Proposed method
Fig. 2 the integration renderings of the each algorithms

By observing each fusion algorithm renderings, we can be seen that proposed algorithm is still better than others. wavelet fusion algorithm is easily affected by background. Document algorithm [9] although can reducing the impact of the background, but it is missing too much background signal. Document algorithm [10] is minimal impact from the visible images, but fused image contrast is too low. It is not conducive to in-depth study of the image.

\section{CONCLUSION}

This paper presents an infrared and visible image fusion algorithm based on characteristic analysis. The algorithm has analysis of infrared and visible images, and using these characteristics to enhance the integration process. According to the regional similarity to classification fusion processing at last. Through experiments, Algorithm can get better fusion compared to other methods. However, this algorithm in terms of speed still exist room for improvement. This will be the focus of future research to study.

\section{REFERENCES}

[1] Tian T, Mei XG, Yu Y, Zhang C, Zhang XY. Automatic visible and infrared face registration based on silhouette matching and robust transformation estimation[J]. INFRARED PHYSICS \& TECHNOLOGY. 2015,69: 145-154

[2]Cui GM, Feng HJ, Xu ZH, Li Q, Chen YT. Detail preserved fusion of visible and infrared images using regional saliency extraction and multi-scale 
image decomposition[J]. OPTICS COMMUNICATIONS. 2015, 341: 199-209.

[3] Xu L, Du JP, Zhang ZH. Infrared-visible video fusion based on motion-compensated wavelet transforms[J]. IET IMAGE PROCESSING. 2015,9(4): 318-328

[4] Yan X, Qin HL, Li J, Zhou HX, Zong JG, Zeng QJ. Infrared and visible image fusion using multiscale directional nonlocal means filter[J]. APPLIED OPTICS. 2015,54(13): 4299-4308.

[5]Liu ZD, Yin HP, Fang B, Chai Y. A novel fusion scheme for visible and infrared images based on compressive sensing[J]. OPTICS COMMUNICATIONS.2015,335: 168-177.

[6]Kong WW, Lei Y, Zhao HX. Adaptive fusion method of visible light and infrared images based on non-subsampled shearlet transform and fast non-negative matrix factorization[J]. INFRARED PHYSICS \& TECHNOLOGY. 2014,67: 161-172

[7]Feng X, Li C, Hu KQ. Infrared and visible image fusion based on deep Boltzmann model[J]. ACTA PHYSICA SINICA. 2014,63(18): 112-115

[8]Gao SS, Jin WQ, Wang LX. Objective color harmony assessment for visible and infrared color fusion images of typical scenes[J]. OPTICAL ENGINEERING. 2012,51(11): 162-167

[9]Xiang TZ, Yan L, Gao RR. A fusion algorithm for infrared and visible images based on adaptive dual-channel unit-linking PCNN in NSCT domain [J]. INFRARED PHYSICS \& TECHNOLOGY. 2015,69: 53-61.

[10]Lu XQ, Zhang BH, Zhao Y, Liu H, Pei HQ. The infrared and visible image fusion algorithm based on target separation and sparse representation[J]. INFRARED PHYSICS \& TECHNOLOGY. 2014,67: $397-407$ 